\title{
Ethical Orientation to Conduct Research Involving Human Participants During COVID-19 Outbreak
}

As chair of the Institutional Review Board (IRB) of University of Costa Rica, I have been asked on several occasions about recommendations to carry out biomedical and non-biomedical research with human beings, in the face of the emergency due to the coronavirus disease 2019 (COVID-19) global pandemic. The use of virtual platforms to request informed consent / assent, conducting virtual interviews or conducting virtual focus groups, have been the most frequent concerns. It is important to clarify this, since the possibility of using digital media instead of fieldwork, implies that there are no delays in the progress of ongoing and new research projects.

Investigators are responsible for ensuring the rights, safety, and well-being of all participants in both biomedical and non-biomedical research. In addition, investigators should comply with the core ethical principles of a) respecting the dignity of people, b) beneficence (do good), c) nonmaleficence (do not harm), d) autonomy e) distributive justice and f) precaution (1). It is imperative to do research with the highest ethical standards. All the basic or technical guidelines of conduct to reduce the risk of transmission of COVID-19 issued by the World Health Organization (WHO), as well as those established by different government health agencies or authorities, must be met to recruit participants, conduct the study, and monitoring. In the case of the University of Costa Rica, it is recommended to follow the guidelines issued by the Ministry of Health of Costa Rica, as well as protocols to mitigate potential risk of transmission developed by the different academic units, research centers and institutes of research.

As the WHO declares "ethical standards for research, remain in effect during public health emergencies" (2). Therefore, it is a fundamental ethical requirement to obtain informed consent in all investigations carried out during emergencies involving human beings, when obtaining biological samples for research purposes, or collecting identifiable data from participating persons. It is obligatory for researchers to inform potential and already recruited participants, how their samples or data are going to be collected, stored, used now or in the future. Participants have the right to consent or decline biological material and data sharing.

It is valid to take informed consent using electronic means, through videos or interactive interfaces. In order for the researcher to ensure that a participant understood the informed consent, there may be interaction through a virtual platform, by telephone or even administer an informed consent through a link and within the document, other links with additional information. One possibility when signing the informed consent form is for participants to sign the document on paper or with a digital signature and send it by email or by ground mail. In some exceptional cases, if this is not possible, as isolation of participants may make this process difficult, when the recommendation for social distancing ends, participants may be asked to sign the document.

RAMIREZ K., 2021: Ethical Orientation to Conduct Research Involving Human Participants During COVID-19 Outbreak.-ODOVTOS-Int. J. Dental Sc., 23-1 (January-April): 8-9. 
Another option is to administer a consent over a monitored or recorded call or video call. Specifically in a video call, if a sensitive topic will be discussed, it is recommended to take the consent in a file and the interview or activity to be developed in another file (and save only the audio of the interview or activity). The way of administration of the informed consent must be previously authorized by the IRB. Depending on the circumstances, the IRB may also decide to waive the requirement to obtain informed consent when it is not feasible to obtain it, the investigation has a significant social value, or when the research only poses minimal risks to participants (3).

When the study is embarked, it is feasible to use and propose alternative methods to obtain results, so that the participants do not have to go to the research site. These alternative methods include phone calls for surveys, interviews, data collection, contact through virtual platforms such as Z00M or TEAMS. Telemedicine can also be used as a way of caring for research participants. Health Insurance Portability and Accountability Act compliant video-conferencing tools are available for researchers. The protocol should make clear the possible risks of conducting interviews, questionnaires, focus groups or other activities in virtual mode, especially when working with vulnerable populations.

Finally, all of these recommendations may also apply to studies that are being carried out. All changes in the research methodology must be documented in the study protocol by an amendment. The timeline must be updated to determine the duration of the study with the change or changes proposed and the impact of these changes on study participants. If necessary, the informed consent/assent should also be updated. All of these matters should be evaluated by the IRB.

\section{REFERENCES}

1. Guidelines research ethics review committee available at: https://vinv.ucr.ac.cr/es/comite-eticocientifico

2. Ethical standards for research during public health emergencies: Distilling existing guidance to support COVID-19 R\&D available at: https:/www.who.int/blueprint/priority-diseases/key-action/ liverecovery-save-of-ethical-standards-for-research-during-public-health-emergencies.pdf

3. COMUNICADO 2: Recomendaciones para realizar investigación biomédica durante el periodo de la emergencia sanitaria por COVID-19 en Costa Rica, available at : https://www.ministeriodesalud. go.cr/index.php/consejos/conis

Disclosure of interest: Opinions are my own and not the views of my employer.

Karol Ramirez DDS, MSC, PhD

Cathedratic/Full Professor Universidad de Costa Rica, Costa Rica.

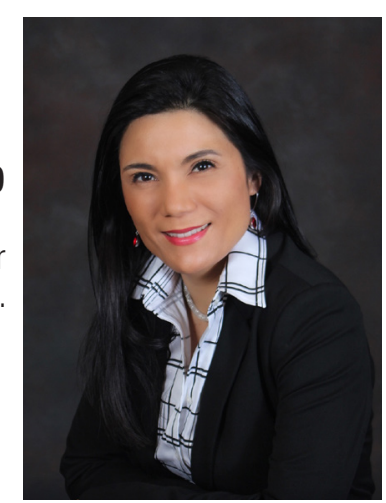

